\title{
Microscopic details of stripes and bubbles in the quantum Hall regime
}

\author{
Josef Oswald ${ }^{1}{ }^{1, *}$ and Rudolf A. Römer ${ }^{2,3,4, \dagger}$ \\ ${ }^{1}$ Institut für Physik, Montanuniversität Leoben, Franz-Josef-Strasse 18, 8700 Leoben, Austria \\ ${ }^{2}$ Department of Physics, University of Warwick, Coventry CV4 7AL, United Kingdom \\ ${ }^{3}$ CY Advanced Studies and LPTM (UMR8089 of CNRS), CY Cergy-Paris Université, F-95302 Cergy-Pontoise, France \\ ${ }^{4}$ Department of Physics and Optoelectronics, Xiangtan University, Xiangtan 411105, Hunan, China
}

(Received 15 January 2020; accepted 31 August 2020; published 28 September 2020)

\begin{abstract}
We use a fully self-consistent laterally resolved Hartree-Fock approximation for numerically addressing the electron configurations at higher Landau levels in the quantum Hall regime for near-macroscopic sample sizes. At low disorder we find spatially resolved, stripe- and bubblelike charge-density modulations and show how these emerge depending on the filling factor. The microscopic details of these boundary regions determine the geometrical boundary conditions for aligning the charge-density modulation either as stripes or bubbles. Transport is modeled using a nonequilibrium network model giving a pronounced anisotropy in the direction of the injected current in the stripe regime close to half filling. We obtain a stripe period of 2.9 cyclotron radii. Our results provide an intuitive understanding of its consequences in strong magnetic fields and indicate the dominance of many particle physics in the integer quantum Hall regime.
\end{abstract}

DOI: 10.1103/PhysRevB.102.121305

In May 2019, the quantum Hall effect [1,2] was formally included among the select group of high-precision experiments to form the basis of a new SI system of units based on the "Planck constant $h$, the elementary charge $e$, the Boltzmann constant $k$, and the Avogadro constant $N_{A}$ " [3]. This had long been awaited and certainly represents a great achievement and fitting fulfillment of the vision for "natürliche Masseinheiten" [4] proposed by Max Planck [5]. In his essay to celebrate this achievement [3], von Klitzing also points out "that a microscopic picture of the quantum Hall effect for real devices with electrical contacts and finite current flow is still missing." Prominent examples of such microscopic details are so-called "bubble" and "stripe" phases [6]. They have been identified, e.g., by transport experiments in higher Landau levels (LLs) of ultrahigh mobility samples [7-9] and are characterized by strong transport anisotropies (stripes) or reentrance effects (bubbles). It is believed that the phases correspond to density modulations with characteristic geometric nonuniformities due to the interplay of Coulomb interaction and the wave functions in higher Landau levels.

Early work in modeling density modulations in the quantum Hall regime, starting from the celebrated Chklovskii, Shklovskii, and Glazman picture [10], assumed unidirectional charge-density waves (CDWs) $[11,12]$ while mean-field treatments established the possibility of anisotropic phases in a Fermi liquid $[13,14]$. However, spatially resolved information does not yet exist of these phases. Experimentally this is due to the intrinsic challenge of using local scanning probes in low temperatures for such remotely doped systems [15-17].

\footnotetext{
*Josef.Oswald@unileoben.ac.at

${ }^{\dagger}$ R.Roemer@warwick.ac.uk
}

Nevertheless, much indirect experimental evidence for the existence of bubble and stripe phases has now been accumulated [17-28]. Theoretical modeling has likewise concentrated on transport signatures of these phases [29-32] while spatially resolved models of bubbles and stripes are only available in clean systems [33]. The period of the stripe patterns has been predicted to follow $d \propto R_{C}$ with $R_{C}=l_{B} \sqrt{2 n+1}$ the cyclotron radius in LL $n$ at the Fermi energy $[11,34]$ and $l_{B}=\sqrt{\hbar / e B}$ the magnetic length. Experimentally, $1.5 R_{c}$ [17] and 3.6 $R_{c}[18]$ have been reported while $\sim 2.7 R_{c}$ is predicted theoretically [34].

In the present work, we show how stripes and bubbles emerge at weak disorder as self-consistent solutions of the Hartree-Fock (HF) equations, i.e., in the experimentally relevant regime and without any ad hoc assumptions beyond a smooth disorder. We provide the full spatial resolution of both phases from the length scale of $l_{B}$ to near macroscopic sample sizes. This high resolution allows quantitative comparison with current experimental efforts [17,18,24,26,27]. A central insight provided by our work is the importance of many-body aspects. It should be clear that a fully self-consistent HF approach in a disordered environment goes well beyond earlier Thomas-Fermi-based (non-)linear screening models. The inclusion of a converged exchange interaction term essentially alters the physics. This not only changes the spatial distribution of stripes and bubbles, but rather is the main reason for their emergence: Neither pure Hartree nor a noninteracting model leads to emerging stripes/bubbles unless coupled with additional assumptions. The key mechanism is a Hund's rule behavior for the occupation of the spin-split LLs. The resulting $g$-factor enhancement is then a local quantity depending on the local filling factor $v(\vec{r})=2 \pi l_{B}^{2} \rho(\vec{r})[35,36]$ with $\rho(\vec{r})$ the local carrier density. This exchange-enhanced $g$ factor is a concept that allows one to discuss the exchange interaction 
(a)

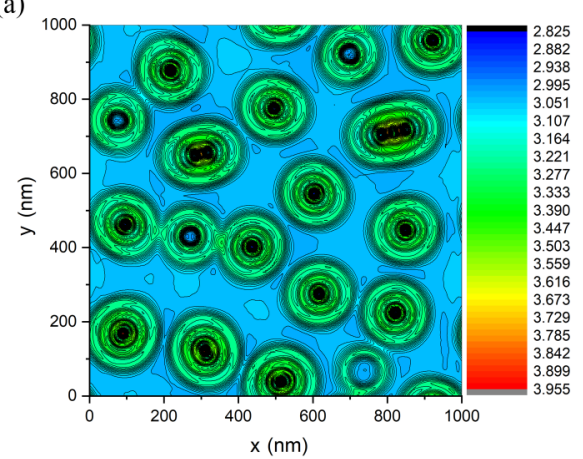

(b)

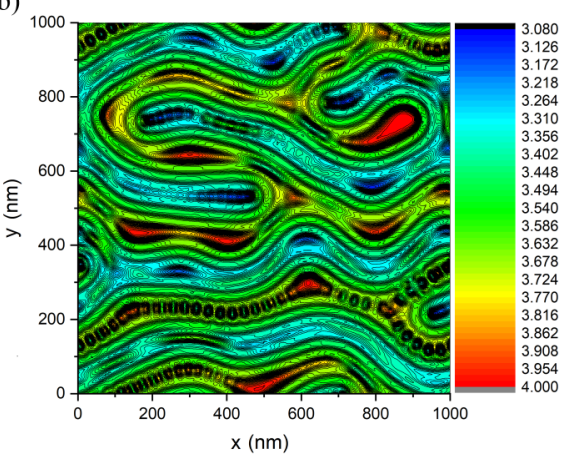

(c)

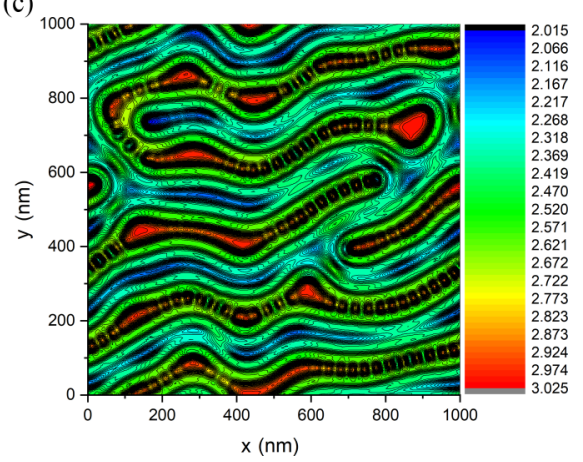

FIG. 1. Spatially resolved filling factor distribution $v_{\downarrow}(\vec{r})$ for different total filling factors (a) $v=6.20$, (b) $v=6.54$, and (c) $v=4.54$ with $B=1.5 \mathrm{~T}$. The colors denote different $v_{\downarrow}(\vec{r})$ values as indicated in the legends. The thin black lines are contours.

within an effective single-electron picture [37,38]. By doing so, we find that the local variation of the enhancement of the Zeeman energy due to $v(\vec{r})$ has to be considered in addition to the laterally varying Hartree potential, leading to a modified effective potential for the electrons that also strongly modifies the screening behavior. In addition to the largely repulsive Hartree part of the self-consistent Thomas-Fermi screening, the $v(\vec{r})$ dependence of enhanced Zeeman energy leads to a positive feedback loop in the self-consistent carrier redistribution and produces an instability of the electron density $\rho$, which may lead to jumps either to a locally full or locally empty LL [35,36], resulting in a clustering of the filling factor that is triggered by the disorder or edge potential. The boundaries of those clusters finally create narrow channels that align mainly along the edge or random potential fluctuations. In the case of a very clean high mobility electron system such a trigger effect for the cluster formation by the random potential is missing and the electron system has to find such a cluster structure by self-organization, resulting in the formation of stripes or bubbles.

The numerical simulations are performed as described in Refs. [35,36,39] via a variational minimization of the self-consistent Hartree-Fock-Roothaan equation [40-43]. To simulate ultrahigh mobility samples, the random potential strength is kept low. The filling factor and the lateral system size are made as large as possible with respect to the available computing power. Configurations up to $1 \mu \mathrm{m}^{2}$ are achievable as shown in Fig. 1 with spatial resolution of $\sim 4.4 \mathrm{~nm}$ well below $l_{B}$ for a magnetic field $B$ varying from, e.g., 1 $\left(l_{B} \sim 26 \mathrm{~nm}\right)$ to $6.5 \mathrm{~T}\left(l_{B} \sim 10 \mathrm{~nm}\right)$. The random potential is generated by Gaussian impurity potentials of radius $40 \mathrm{~nm}$, the number of impurities is $N=2000$, and their random placement results in a fluctuating potential of $V_{\max }=0.43 \mathrm{mV}$ and $V_{\min }=-0.50 \mathrm{mV}$ [44]. At a total filling factor of, e.g., $v=6.54\left(=v_{\downarrow}+v_{\uparrow}=3.54+3.0\right)$ and $B=1.5 \mathrm{~T}$, this corresponds to more than 2000 electrons. In order to generate transport data, we employ a nonequilibrium network model (NNM) introduced previously [45]. A very large number of step by step calculations are required [46] in the NNM. Hence, for keeping within the available computing time, the transport simulations have been performed for smaller sample size such as $500 \times 500 \mathrm{~nm}^{2}$.

In Fig. 1 we depict the variation in $v_{\downarrow}(\vec{r})$ for three different densities at fixed magnetic field. Figure 1(a) shows the situation far from any half-odd average $v_{\downarrow}$. The required area of the filled (spin-down) clusters is only a minor part of the total area. As seen in the figure this can be achieved by a nearly evenly spaced distribution of bubblelike-shaped clusters. Since we are considering already the fourth partly filled spin-split LL, the boundaries of the bubbles consist of three substripes because of the three nodes of the Landau basis function for the fourth LL [16]. A boundary with such an internal structure takes up a substantial area as well and even tends to dominate the region of a single bubble as a whole. In other words, the area that one bubble needs is dominated by the width of the boundaries and not by the region of an assumed idealized full LL. When increasing the filling factor towards half filling, as shown in Fig. 1(b), it becomes clear that such a bubblelike geometry of the clusters is impossible to achieve with the given total $\nu$, because round bubbles would leave too much unfilled space between the bubbles even if touching each other. The only way to remove the unfilled space between bubbles is a change of the geometry so that the boundaries of different clusters arrange almost in parallel, which means a transfer to a stripelike geometry. In order to demonstrate that the width of the boundaries of the stripes and bubbles depend on the LL index, Fig. 1(c) shows the half-filled LL at filling factor $v \approx 4.5$ instead of $v=6.5$ and one can see that there is one substripe less at the boundary, allowing a more dense arrangement of stripes or bubbles than in the higher LLs. These results suggest that the higher the filling $v$ the higher the tendency for creating the stripe pattern near a half-filled LL. Overall, the figures show that our HF calculations can provide a fascinating insight into the spatial behavior of bubble and stripe configurations.

Figure 2 contains the transport data obtained by applying the NNM at different carrier densities. Since the stripe alignment tends to be more horizontal, i.e., along the $x$-direction as shown in Fig. 1, the longitudinal resistance $R_{x x}$ appears higher for vertical sample current and lower for horizontal current flow [44]. This is consistent with experimental observations resulting in large $R_{x x}$ peaks for vertical current, while for a horizontal current the $R_{x x}$ peaks are hardly visible. When the difference between horizontal and vertical $R_{x x}$ is no longer prominent, we find that we have reached a bubble phase.

The characteristics of the microscopic structure of the stripes consist on one hand in the periodicity of the stripe pattern and on the other hand in the microscopic details of the 


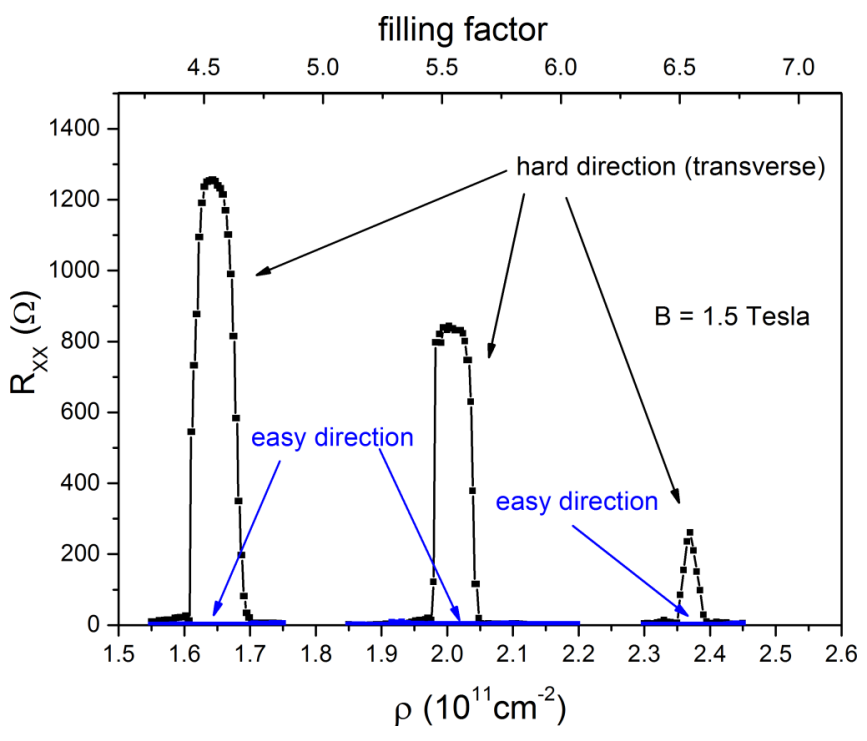

FIG. 2. Longitudinal resistance $R_{x x}$ for easy (horizontal) and hard (vertical) directions (cf. Fig. 1) as a function of carrier density $\rho$. The sample size is $500 \times 500 \mathrm{~nm}^{2}, B=1.5 \mathrm{~T}$, the impurity radius $40 \mathrm{~nm}$, and the number of impurities $N=100$. The random impurity placement results in a fluctuating potential from $V_{\min }=-1.7 \mathrm{mV}$ to $V_{\max }=1.7 \mathrm{mV}$.

boundaries of the stripes. Together with the geometric shape of the stripes (and, indeed, the bubbles), these determine the relation between the areas of full and empty LLs. Focusing first on the periodicity, Fig. 3 presents the two-dimensional Fourier transformation of the lateral carrier density at various magnetic fields from $B=1 \mathrm{~T}$ to $B=6.5 \mathrm{~T}$ at a fixed filling factor of $v=4.5$. While Fig. 3(a) shows only a selection of a few spectra, Fig. 3(b) displays the trend of all evaluated spectra. As can be seen in the $x$ direction there is a smooth and somewhat smeared out distribution that starts from zero, indicating that there is no clear periodicity in the $x$ direction. In contrast, in the $y$ direction, there is a well pronounced maximum which matches the reciprocal stripe period in the $y$ direction as also seen in Figs. 1(b) and 1(c). For $B=1.5 \mathrm{~T}$ the reciprocal period (wave number) appears to be close to 6 [47] and for $B=6 \mathrm{~T}$ it matches 12 , which is consistent with a $\sqrt{B}$ dependence. Furthermore, we extract the mean period of the corresponding stripe patterns, similar to those shown in Fig. 1, to be approximately $175 \mathrm{~nm}$ for $B=1.5 \mathrm{~T}$ and $81 \mathrm{~nm}$ for $B=6 \mathrm{~T}$. Extending the analysis to other $B$ values, we indeed find a clear $\sqrt{B}$ behavior as shown in Fig. 3(b). Previous theoretical results on the period $d$ of the stripes have led to the expectation $d=\alpha R_{c}=\alpha \sqrt{(2 n+1) \hbar B / e}[11,34]$. Experimentally, $\alpha=1.5$ [17] and 3.6 [18] have been estimated. For $n=3$ and $B=1.5 \mathrm{~T}$, these give 88 and $210 \mathrm{~nm}$, respectively. Obviously, only the latter one is compatible with our result at $1.5 \mathrm{~T}$. Conversely, from Fig. 3(b), we extract a value $\alpha=2.9 \pm 0.1$. This agrees very well with previous straight-line CDW-based predictions of 2.7 [34] and 2.8 [12].

Friess et al. [17] investigated the Knight shift in nuclear magnetic resonance (NMR) spectra to demonstrate the coexistence of regions with different spin polarization due to the periodic variation of the filling factor in stripe and bubble phases. Their method provides direct information about the
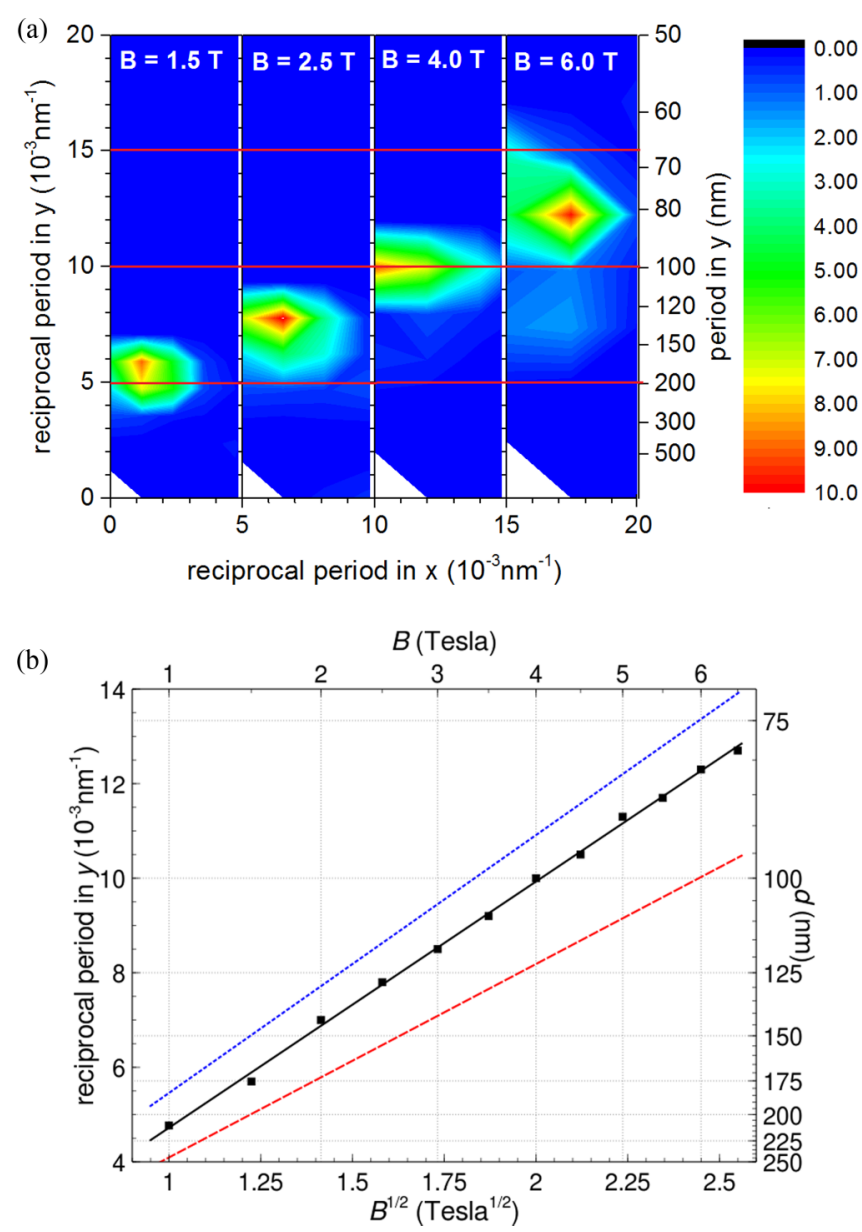

FIG. 3. (a) Two-dimensional Fourier spectra at $v=4.5$ for different $B$ fields. The different spectra are shifted to the right for better visibility. For a sample size of $1000 \times 1000 \mathrm{~nm}^{2}$, the frequency in units of $0.001 \mathrm{~nm}^{-1}$ (left axis) equals the number of stripes that can be accommodated within the boundaries. The corresponding period length is shown on the right axis and the color bar indicates the Fourier intensity in arb. units. The horizontal red lines indicate selected values. (b) Variation of the reciprocal stripe period $1 / d(\boldsymbol{\square})$ as a function of $\sqrt{B}$. The solid lines correspond to $d=\alpha \sqrt{2 n+1} R_{c}$ for the LL index $n=3$ with $\alpha=2.7$ (blue line, theory) [34], 3.6 (red line, experiment) [18], and 2.9 (black line, our fit), respectively. The gray dotted grid lines highlight selected $B$ and $d$ values.

area fractions, although there is no direct information about geometry and periodicity. In order to extract also microscopic details of the structural information, they use a semiclassical model $[17,48]$ based on superpositions of the single-electron densities obtained from the Landau basis functions. In our case, we can similarly model the NMR intensity as $I_{v}(f)=$ $\left.\int \mathcal{G}\left\{f-\left[f_{0, v-1}-v(\vec{r}) K_{\max , v}\right]\right\}\right) d r^{2}$ with Gaussian $\mathcal{G}$ describing the absorption spectrum of individual nuclei [48], $f$ the NMR frequency, $K_{\max }$ the maximal Knight shift for the fully spin-polarized LL at odd $\nu$, and $f_{0}$ the frequency of the nonshifted NMR line for the non-spin-polarized situation at even filling factor $v-1$. Numerically, $I_{v}(f)$ is calculated by evaluating our interacting $v(\vec{r})$ at each $\vec{r}$ and summing over all points of a typically $229 \times 229$ grid. In Fig. 4, we show that $I_{v}(f)$ exhibits features that redshift to lower frequencies 
(a)

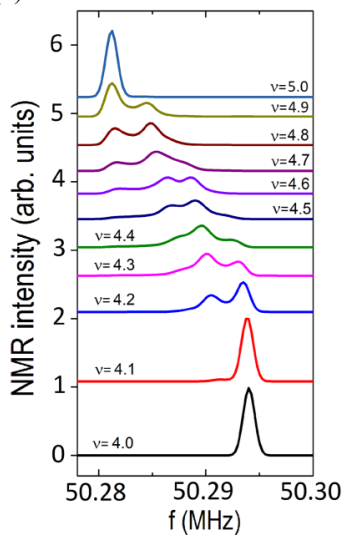

(b)

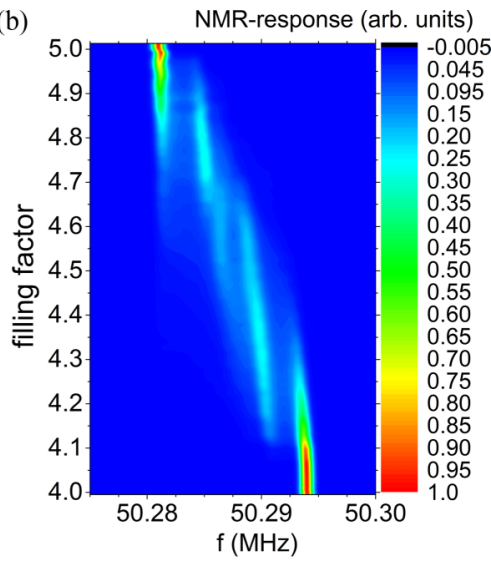

FIG. 4. (a) Simulation results of $I_{v}(f)$ using our HF results for $v_{\downarrow}(\vec{r})$ as input. The traces are shifted vertically for better visibility and varied in steps of $\Delta v=0.1$. (b) Color plot of $I_{v}(f)$ for very fine resolution $\Delta v=0.01$. The color legend gives the numerical values of $I_{v}(f)$.

when increasing $v$, e.g., from the non-spin-polarized $v=4$ to a fully spin polarized $v=5$ (see Supplemental Material [44] for $v=2 \rightarrow 3$ ). At intermediate filling factors the spectral response splits, mainly into double/triple peak structures. This indicates the coexistence of regions with different filling factor as expected from a stripe- or bubble-like electron distribution (see Supplemental Material [44]). Around $v \sim 4.4$ one can recognize three peaks with changing weights in the superposition while varying the total filling factor. This is in good agreement with the experimental results [17], but naturally less so when compared to a single-particle modeling.

Intuitively, the driving force for the formation of bubbles and stripes can be understood as follows: The Hund's rule behavior causes a $g$-factor enhancement that maintains the tendency to fill up just one spin level as much as possible before starting to fill up the next spin level. When entering a stripe/bubble from outside (e.g., $v=4$, not spin polarized) to inside (e.g., $v=5$, fully spin polarized) the $g$-factor enhancement also drastically increases. Inside the stripe/bubble the spin levels are strongly pushed apart, corresponding to a significantly lower energy of the occupied lower spin state, while outside there is minimal $g$-factor enhancement at even filling factor $v=4$. In terms of an effective single-particle picture the electrons in the stripes/bubbles encounter a potential well established by the local variation of the $g$-factor enhancement and which dominates over the repulsive Hartree interaction. From a qualitative point of view, the stripes can be understood as leaky noncoupled electron waveguides that consist of self-assembled one-dimensional potential wells and the leakage shows up as the boundary region as discussed before.

In conclusion, we have computed the microscopic picture of stripe and bubble phases for the integer quantum Hall (IQH) regime at high LLs and in weak disorder. Our results rely on spatially resolved, self-consistent HF calculations of nearly macroscopic sizes of $O\left(1 \mu \mathrm{m}^{2}\right)$ and, for transport calculations, are coupled to device contacts with finite currents. The existence of microscopic stripes and bubbles at weak disorder is thus confirmed. Their spatial features show intriguing extended oscillations along the stripes and surrounding the bubbles. These are clearly due to the structure of the underlying Landau states. We find that the stripe period scales with $B^{-1 / 2}$ as expected and agrees in detail with previous experimental measurements. Overall, together with results from $\mathrm{HF}$ calculations in the strong disorder regime $[35,36,39]$, this shows that the IQH regime can now be described spatially resolved with high accuracy from microscopic to near-macroscopic length scales. Our results shine a light on the understanding of the microscopic picture of the IQH effect and demonstrate the permanent dominance of many-particle physics for quantum Hall physics. The demonstrated Hund's rule behavior in context with the $g$-factor enhancement allows one to incorporate the exchange interaction into an intuitive understanding of the major effects driving weakly and strongly disordered quantum Hall systems.

The data accompanying this publication are available from the corresponding authors.

This work received funding from the CY Initiative of Excellence (Grant "Investissements d'Avenir" ANR-16IDEX-0008) and developed during R.A.R.'s stay at the CY Advanced Studies, whose support is gratefully acknowledged. We thank Warwick's Scientific Computing Research Technology Platform for computing time and support. J.O. thanks Karl Flicker for valuable technical support for the MUL high performance cluster in Leoben, where the Hartree-Fock calculations have been performed.
[1] K. v. Klitzing, G. Dorda, and M. Pepper, Phys. Rev. Lett. 45, 494 (1980).

[2] D. C. Tsui, H. L. Stormer, and A. C. Gossard, Phys. Rev. Lett. 48, 1559 (1982).

[3] K. von Klitzing, Phys. Rev. Lett. 122, 200001 (2019).

[4] Translation: Natural measurement units.

[5] M. Planck, Ann. Phys. 306, 69 (1900).

[6] M. M. Fogler, in High Magnetic Fields, edited by C. Berthier, L. P. Levy, and G. Martinez (Springer, Berlin/Heidelberg, 2002), Vol. 595, pp. 98-138.
[7] M. P. Lilly, K. B. Cooper, J. P. Eisenstein, L. N. Pfeiffer, and K. W. West, Phys. Rev. Lett. 82, 394 (1999).

[8] R. Du, D. Tsui, H. Stormer, L. Pfeiffer, K. Baldwin, and K. West, Solid State Commun. 109, 389 (1999).

[9] R. Du, W. Pan, H. Stormer, D. Tsui, L. Pfeiffer, K. Baldwin, and K. West, Phys. E (Amsterdam, Neth.) 6, 36 (2000).

[10] D. B. Chklovskii, B. I. Shklovskii, and L. I. Glazman, Phys. Rev. B 46, 4026 (1992).

[11] A. A. Koulakov, M. M. Fogler, and B. I. Shklovskii, Phys. Rev. Lett. 76, 499 (1996). 
[12] M. M. Fogler, A. A. Koulakov, and B. I. Shklovskii, Phys. Rev. B 54, 1853 (1996).

[13] E. Fradkin and S. A. Kivelson, Phys. Rev. B 59, 8065 (1999).

[14] B. Spivak and S. A. Kivelson, Ann. Phys. 321, 2071 (2006).

[15] K. Hashimoto, C. Sohrmann, J. Wiebe, T. Inaoka, F. Meier, Y. Hirayama, R. A. Römer, R. Wiesendanger, and M. Morgenstern, Phys. Rev. Lett. 101, 256802 (2008).

[16] K. Hashimoto, T. Champel, S. Florens, C. Sohrmann, J. Wiebe, Y. Hirayama, R. A. Römer, R. Wiesendanger, and M. Morgenstern, Phys. Rev. Lett. 109, 116805 (2012).

[17] B. Friess, V. Umansky, L. Tiemann, K. von Klitzing, and J. H. Smet, Phys. Rev. Lett. 113, 076803 (2014).

[18] I. V. Kukushkin, V. Umansky, K. von Klitzing, and J. H. Smet, Phys. Rev. Lett. 106, 206804 (2011).

[19] Y. Liu, D. Kamburov, M. Shayegan, L. N. Pfeiffer, K. W. West, and K. W. Baldwin, Phys. Rev. B 87, 075314 (2013).

[20] W. Pan, A. Serafin, J. S. Xia, L. Yin, N. S. Sullivan, K. W. Baldwin, K. W. West, L. N. Pfeiffer, and D. C. Tsui, Phys. Rev. B 89, 241302(R) (2014).

[21] X. Wang, H. Fu, L. Du, X. Liu, P. Wang, L. N. Pfeiffer, K. W. West, R.-R. Du, and X. Lin, Phys. Rev. B 91, 115301 (2015).

[22] J. Pollanen, K. B. Cooper, S. Brandsen, J. P. Eisenstein, L. N. Pfeiffer, and K. W. West, Phys. Rev. B 92, 115410 (2015).

[23] M. E. Msall and W. Dietsche, New J. Phys. 17, 043042 (2015).

[24] M. A. Mueed, M. S. Hossain, L. N. Pfeiffer, K. W. West, K. W. Baldwin, and M. Shayegan, Phys. Rev. Lett. 117, 076803 (2016).

[25] Q. Shi, M. A. Zudov, Q. Qian, J. D. Watson, and M. J. Manfra, Phys. Rev. B 95, 161303(R) (2017).

[26] B. Friess, Y. Peng, B. Rosenow, F. von Oppen, V. Umansky, K. von Klitzing, and J. H. Smet, Nat. Phys. 13, 1124 (2017).

[27] B. Friess, V. Umansky, K. von Klitzing, and J. H. Smet, Phys. Rev. Lett. 120, 137603 (2018).

[28] K. Bennaceur, C. Lupien, B. Reulet, G. Gervais, L. N. Pfeiffer, and K. W. West, Phys. Rev. Lett. 120, 136801 (2018).

[29] A. M. Ettouhami, C. B. Doiron, F. D. Klironomos, R. Côté, and A. T. Dorsey, Phys. Rev. Lett. 96, 196802 (2006).

[30] A. M. Ettouhami, C. B. Doiron, and R. Côté, Phys. Rev. B 76, 161306(R) (2007).
[31] R. Côté, H. A. Fertig, J. Bourassa, and D. Bouchiha, Phys. Rev. B 66, 205315 (2002).

[32] R. Côté and A. M. Simoneau, Phys. Rev. B 93, 075305 (2016).

[33] R. Côté, C. B. Doiron, J. Bourassa, and H. A. Fertig, Phys. Rev. B 68, 155327 (2003).

[34] M. O. Goerbig, P. Lederer, and C. M. Smith, Phys. Rev. B 69 , 115327 (2004).

[35] J. Oswald and R. A. Römer, Europhys. Lett. 117, 57009 (2017).

[36] J. Oswald and R. A. Römer, Phys. Rev. B 96, 125128 (2017).

[37] S. A. Wiegers, M. Specht, L. P. Lévy, M. Y. Simmons, D. A. Ritchie, A. Cavanna, B. Etienne, G. Martinez, and P. Wyder, Phys. Rev. Lett. 79, 3238 (1997).

[38] E. M. Kendirlik, S. Sirt, S. B. Kalkan, W. Dietsche, W. Wegscheider, S. Ludwig, and A. Siddiki, Sci. Rep. 3, 3133 (2013).

[39] C. Sohrmann and R. A. Römer, New J. Phys. 9, 97 (2007).

[40] H. Aoki, J. Phys. C 12, 633 (1979).

[41] D. Yoshioka and H. Fukuyama, J. Phys. Soc. Jpn. 47, 394 (1979).

[42] A. H. MacDonald and G. C. Aers, Phys. Rev. B 34, 2906 (1986).

[43] A. H. MacDonald and S. M. Girvin, Phys. Rev. B 38, 6295 (1988).

[44] See Supplemental Material at http://link.aps.org/supplemental/ 10.1103/PhysRevB.102.121305 for a different graphical representation of Fig. 1, the absence of stripes and bubbles in $v(\vec{r})$ for pure Hartree interaction and without any interaction, a plot of $R_{x y}$, a graphical representation of the disorder potential, a consistency check of $I_{v}(f)$ for various test patterns, and a plot of $v(\vec{r})$ corresponding to Fig. 4(b).

[45] J. Oswald and M. Oswald, J. Phys.: Condens. Matter 18, R101 (2006).

[46] C. Sohrmann, J. Oswald, and R. A. Römer, in Quantum and Semi-Classical Percolation and Breakdown in Disordered Solids, edited by A. K. Sen, K. K. Bardhan, and B. K. Chakrabarti (Springer, Berlin/Heidelberg, 2009), pp. 1-31.

[47] The scale $1 / 1000 \mathrm{~nm}^{-1}$ of the reciprocal period is chosen in a way that it matches the number of periods appearing on the length of a sample square of $1000 \times 1000 \mathrm{~nm}^{2}$.

[48] L. Tiemann, G. Gamez, N. Kumada, and K. Muraki, Science 335, 828 (2012). 